\title{
A Superlattice Infrared Photodetector Integrated With Multiple Quantum Wells to Improve the Performance
}

\author{
Jen-Hsiang Lu, Kun-Jheng Wu, Kuang-Jou Hsieh, Chieh-Hsiung Kuan, Member, IEEE, Juei-Yang Feng, \\ Tsong-Sheng Lay, Member, IEEE, Chen-Wei Yang, and Shun-Li Tu
}

\begin{abstract}
An infrared photodetector using the structure of a 15-period superlattice (SL) integrated with 50-period multiple quantum wells (MQWs) is investigated. The MQWs are utilized to reduce the noise current power and to add the response range. From the results of current ratio and response, the photocurrent of the SL is not reduced by the additional MQWs but the dark current is. Hence, due to the low noise gain and low dark current, the maximum detectivity $\left(D^{*}\right)$ can occur at low negative bias. In addition, the photovoltaic response even appears at $80 \mathrm{~K}$. It is observed that the photoelectron transport directions from the SL and the MQWs are opposite under zero bias. In comparison with the SL with a single barrier, this structure also demonstrates the higher photocurrent and lower dark current. From our experimental results, this structure is appropriate for the operation at low bias and high temperature. However, the tradeoff is the small operational voltage range.
\end{abstract}

Index Terms-Infrared detectors, noise filter, photovoltaic (PV) detectors, quantum-well devices, superlattice.

\section{INTRODUCTION}

$\mathbf{I}$ N RECENT years, quantum-well infrared photodetectors (QWIPs) have attracted much attention, for example, in the multicolor [1]-[4] and high frequency [5]-[7] detections. For the fabrication of the QWIP-based focal plane array (FPA) system, the high current density and the capacitor saturation of the readout IC (ROIC) are two important issues. Some improvements such as Schottky barrier [8] and the photovoltaic (PV) operation mode [9]-[13] were used to lower the current density. On another hand, we may operate the device under low bias range to achieve the low dark current density but with additional photocurrent to improve the associated low photoresponse. The superlattice (SL) is hence integrated with the multiple quantum wells (MQWs) to achieve the purpose. In comparison with QWIPs, SL infrared photodetectors (SLIPs) have three characteristics including low-voltage operation, broadband photoresponse, and voltage tunability [14]-[16].

Manuscript received March 28, 2006; revised August 3, 2006. This work was supported by the National Science Council of Taiwan, R.O.C., under Contract NSC 94-2215-E-002-017.

J.-H. Lu, K.-J. Wu, K.-J. Hsieh, and C.-H. Kuan are with the Department of Electrical Engineering and Graduate Institute of Electronics Engineering, National Taiwan University, Taipei 10617, Taiwan, R.O.C. (e-mail: kuan@cc.ee. ntu.edu.tw).

J.-Y. Feng and T.-S. Lay are with Graduate Institute of Electro-Optical Engineering, National Sun Yat-Sen University, Kaohsiung 80424, Taiwan, R.O.C.

C.-W. Yang and S.-L. Tu are with Opto Tech Corporation, Hsinchu 30078, Taiwan, R.O.C.

Color versions of Figs. 1 and 3-7 are available online at http://ieeexplore. ieee.org.

Digital Object Identifier 10.1109/JQE.2006.884584
In addition to the photocurrent, the noise reduction is also considered. Generally, the SL is integrated with a single barrier to reduce the dark current, and the corresponding noise characteristic is the shot noise. In this paper, we replace the single barrier with MQWs. They are expected to act as a noise filter to reduce the noise current power and thereby to increase the specific detectivity $\left(D^{*}\right)$.

Fig. 1(a) and (b) are the schematic band diagrams of a SL with MQWs and with a single barrier, respectively. For a SL with a single barrier, the noise is due to thermally assisted tunneling and is given by the shot noise performance [15] as mentioned before

$$
i_{\mathrm{SB}}^{2}=2 e I_{D}
$$

where $i_{\mathrm{SB}}$ is the noise current power spectral density (PSD) of this structure and $I_{D}$ is its dark current.

On the other hand, the noise in MQWs is the generation-recombination noise and is given by [17]

$$
i_{\mathrm{QW}}^{2}=4 e g_{n} I_{D}
$$

where $i_{\mathrm{QW}}$ is the noise current PSD of MQWs, $g_{n}$ is the noise gain of MQWs and $I_{D}$ is dark current of the device.

The equivalent circuit of the structure of a SL and a barrier (the single barrier or MQWs) is sketched in Fig. 1(c), where $i_{\mathrm{SL}}$ and $i_{\text {barrier }}$ are the noise current PSD generated by the SL and the barrier, respectively, and $R_{\mathrm{SL}}$ and $R_{\text {barrier }}$ are the equivalent resistances of the SL and the barrier, respectively. Based on the equivalent small signal noise circuit model [18], the applied dc voltage source in external circuit can be treated as short-circuit, and the total noise current PSD of the structure can be expressed as

$$
i_{n}^{2}=\frac{i_{\mathrm{SL}}^{2} R_{\mathrm{SL}}^{2}+i_{\text {barrier }}^{2} R_{\mathrm{barrier}}^{2}}{\left(R_{\mathrm{SL}}+R_{\mathrm{barrier}}\right)^{2}}
$$

Because $R_{\text {barrier }}$ is much higher than $R_{\mathrm{SL}}, i_{n}^{2}$ can be simplified as $i_{\text {barrier }}^{2}$. Therefore, the noise current PSD can be changed from $2 e I_{D}$ to $4 e g_{n} I_{D}$ by replacing the single barrier with MQWs, and is reduced if $g_{n}$ is less than 0.5 . The noise gain in MQWs is defined by $g_{n}=1 /\left(N p_{c}\right)$ [20], where $N$ is the number of wells, and $p_{c}$ is the capture probability. We design the period number of the MQWs to be 50, and operate the detector under low bias to make the $p_{c}$ be nearly 1 . Therefore, the low noise gain can be obtained under low bias operation. 


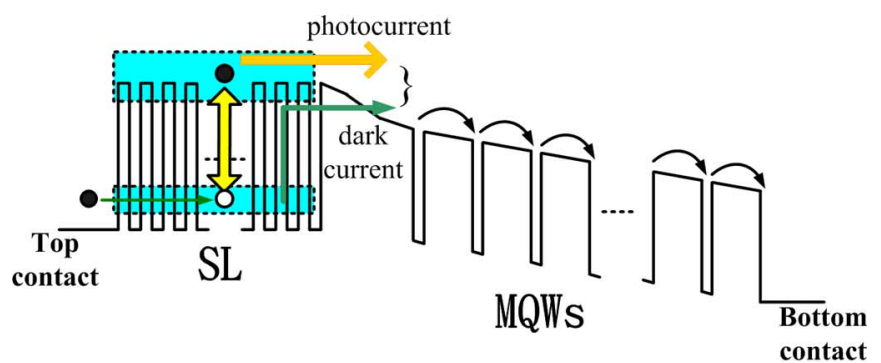

(a)

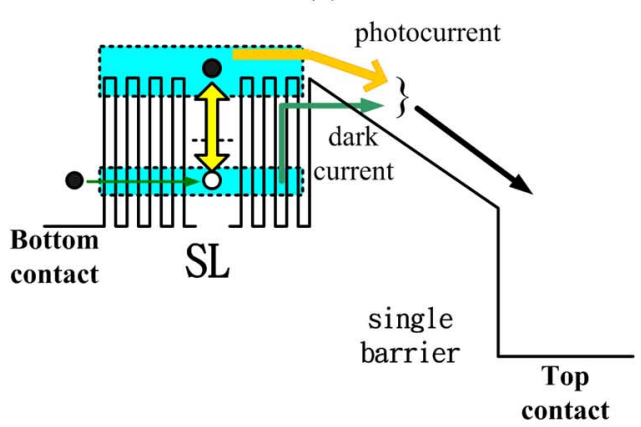

(b)

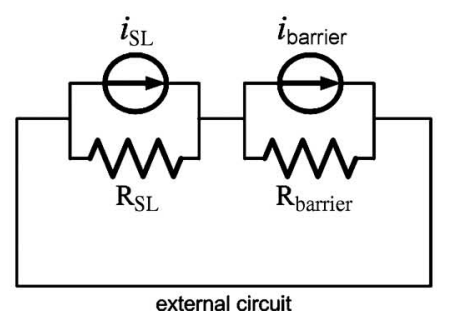

(c)

Fig. 1. (a) Schematic band diagram of Sample A, a SL with MQWs, under low bias. (b) Schematic band diagram of Sample B, a SL with a single barrier. (c) Equivalent circuit of a SL with a barrier.

Due to the high capture probability under low bias, almost all electrons traversing a barrier are recaptured into the neighboring well and produce the capture current. This current must be balanced by the emission current from the well under steady state. This process will repeat till the last quantum well as shown in Fig. 1(a). Therefore, the current flowing through the device is the same as the one injected at the beginning of the device. In our case, the beginning is a SL which is suitable for low-bias operation, so the photocurrent generated from SL can also flow through the whole structure without loss under low bias. On the other hand, the dark current also traverses the same path. In order to decrease the dark current, we will raise the barrier height in the first period to reduce the thermionic emission (TE) and thermally assisted tunneling (TAT) currents. Because the photoelectron's energy is higher, the decrement of photocurrent is less sensitive to the higher barrier.

\section{SAMPle StRUCtURE AND OPERATIONAL MECHANISM}

Two samples with the same 15-period SL but different barriers have been used to investigate the characteristics. Their barriers are MQWs and a single barrier which belong to Sample A and $\mathrm{B}$, respectively.
Sample A contains a top contact layer, a SL, a 60-nm $\mathrm{Al}_{x} \mathrm{Ga}_{1-x}$ As graded barrier $(x=0.29$ for $20 \mathrm{~nm}, x=0.29$ to $x=0.21$ for $20 \mathrm{~nm}$ and $x=0.21$ for $20 \mathrm{~nm}$ ), a 50 -period MQWs and a bottom contact layer. Sample B is a SL with a single barrier sandwiched between two contact layers. The single barrier is a $300 \mathrm{~nm} \mathrm{Al} \mathrm{n}_{0.25} \mathrm{Ga}_{0.75} \mathrm{As}$ layer. Each period of the MQWs consists of $6 \mathrm{~nm}$ GaAs well $\left(n=4 \times 10^{17} \mathrm{~cm}^{-3}\right)$ and $50 \mathrm{~nm} \mathrm{Al}_{0.21} \mathrm{Ga}_{0.79} \mathrm{As}$ barrier (undoped), and each period of the SL consists of $6 \mathrm{~nm} \mathrm{GaAs}$ well $\left(n=4 \times 10^{17} \mathrm{~cm}^{-3}\right)$ and $4 \mathrm{~nm} \mathrm{Al}_{0.29} \mathrm{Ga}_{0.71} \mathrm{As}$ barrier (undoped). The absorption wavelength range of the SL is $6-10 \mu \mathrm{m}$. The transition type of the MQWs is bound-to-bound and its longest absorption wavelength is about $11.7 \mu \mathrm{m}$. The different absorption ranges of the SL and the MQWs are designed for the broadband response. A $45^{\circ}$ facet on the sample substrate is fabricated to allow the TM polarized infrared light to radiate on the photodetector. The positive bias is defined as the top contact layer is positive. The photocurrent spectra are measured by the Fourier transform infrared (FT-IR) spectrometer.

The schematic band diagrams of Sample A under low negative bias and Sample B under positive bias are shown in Fig. 1(a) and (b), respectively. It should be noted that the layer structures of these two samples are opposite. For Sample A under negative bias, photoelectrons from both the SL and the MQWs transport under the electric field, and contribute to the photocurrent. On the other hand, under positive bias, the photoelectrons from the SL and the MQWs move toward the top contact layer. Among them, those photoelectrons from the SL would be supplied by the electrons from the top contact through the first miniband. The electron transport becomes an internal circulation in the SL, which makes no electrons be collected by the external circuit. Therefore, only the MQWs contribute to the photocurrent under positive bias. The operational mechanism of the asymmetric structure has been discussed in our previous work [16]. For Sample B, photoelectrons can be collected only under positive bias as shown in Fig. 1(b).

\section{EXPERIMENTAL RESULTS AND DISCUSSIONS}

\section{A. Noise Gain}

Fig. 2 illustrates the noise gain of Sample A measured under negative bias at $80 \mathrm{~K}$. The detailed noise measurement setup can be found in our previous article [19]. It is observed that the gain is very small under low bias range, which shows agreement with the theoretical value as mentioned before. For example, the noise gain value is about 0.01 at $-0.2 \mathrm{~V}$. Hence, by replacing the single barrier with MQWs, $i_{n}^{2}$ is 50 times less than $i_{\mathrm{SB}}^{2}$ to improve the detectivity which will be discussed later.

\section{B. Photoresponse}

Fig. 3 is the photoresponse of Sample A under $-0.2 \mathrm{~V}$ at different temperatures. Its detection range is compatible with the intensity distribution of $300-\mathrm{K}$ blackbody radiation which is sketched as the dotted line (not in scale). The spectrum can be divided into three parts which come from the different structures as indicated in the figure. The right inset in Fig. 3 shows the response at $80 \mathrm{~K}$ under high negative biases. It is observed that the MQWs response dominates the spectrum under high bias. 


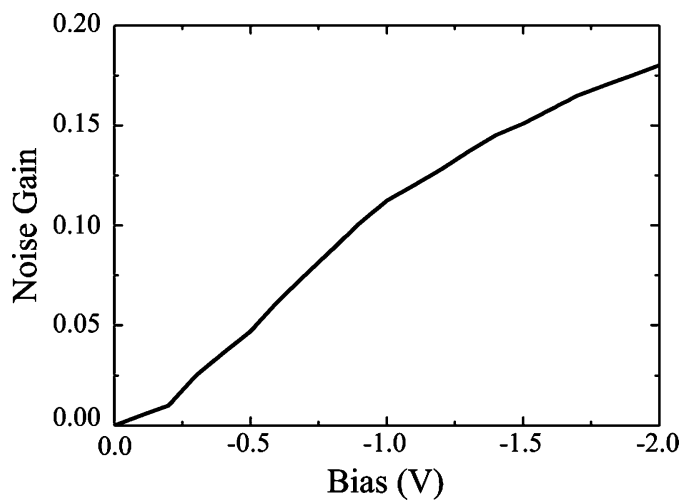

Fig. 2. Noise gain of Sample A under negative bias at $80 \mathrm{~K}$. We observe the gain value is very small under low bias range.

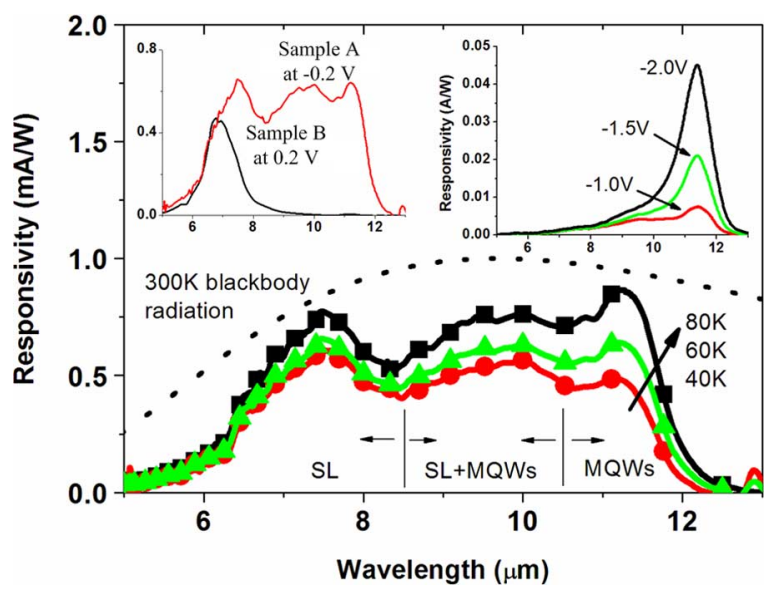

Fig. 3. Photoresponse of Sample A under $-0.2 \mathrm{~V}$ at different temperatures. Its detection range is compatible with the intensity distribution of $300-\mathrm{K}$ blackbody radiation which is sketched as the dotted line (not in scale). The left inset shows the response of Sample A at $-0.2 \mathrm{~V}$ and that of Sample B at $0.2 \mathrm{~V}$. The right inset presents the response of Sample A at $80 \mathrm{~K}$ under high biases. It is observed that the MQWs response dominates the spectrum.

Actually, Sample A has almost the same response under high positive and negative biases.

From the above results, the functions of the MQWs are not only to act as a noise filter but also to enhance the long wavelength response for broadband detection. Generally, the spectral width $\Delta \lambda / \lambda$ of a bound-to-continuum QWIP is about 30\% [20], while Sample A's $\Delta \lambda / \lambda$ can be higher than $50 \%$ under -0.2 to $-0.5 \mathrm{~V}$.

The left inset in Fig. 3 presents the response of Sample A at $-0.2 \mathrm{~V}$ and that of Sample B at 0.2 V. Despite the smaller effective electric field across Sample A due to the MQWs thickness, the responsivity of Sample A is higher than that of Sample B. Their response difference is attributed to the barrier scattering effect. The electrons transporting through the barrier will suffer from the scattering which leads to the decrement of response. The thinner barrier can allow electrons to cross it with minimum chance for scattering. As shown in Fig. 1(a), though the total thickness of Sample A is thicker, the electrons can traverse only one barrier each time under low bias, and the MQWs can be seen as 50 identical single barriers in series. Except the first graded

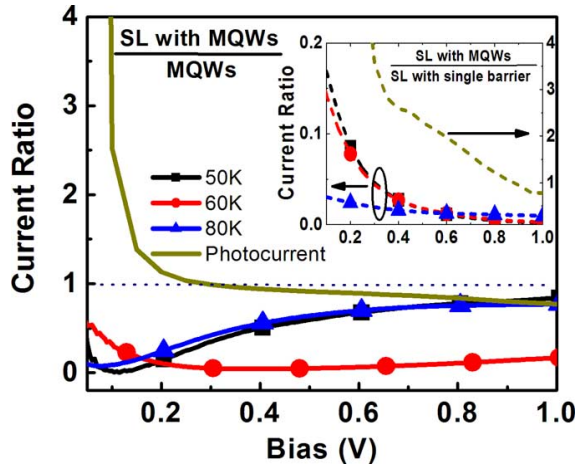

Fig. 4. Solid and dashed lines show the current ratio of the SL with the MQWs over the MQWs and over the SL with a single barrier, respectively. The lines with and without symbols are the dark current and photocurrent ratio, respectively. The SL with the MQWs demonstrates the higher photocurrent but lower bark current.

barrier, the equivalent single barrier width is $50 \mathrm{~nm}$ which is thinner than the single barrier in Sample B and makes the electron transport more likely to be ballistic to produce the higher response in Sample A.

It is noteworthy in Fig. 3 that the responsivity is enhanced with temperature, which is caused by some photoelectrons tunnel through the barrier with the assistance of the thermal energy. This mechanism is similar to the TAT dark current. The detailed physical mechanism will be studied in our future paper.

\section{Current Ratio Versus Bias}

The solid lines in Fig. 4 show the ratio of either the dark current or the photocurrent in Sample A under negative bias over that under positive bias. That is the current ratio of the SL with the MQWs over only the MQWs. In order to compare the effect of the barrier structure, the dashed lines in the inset demonstrate the ratio of current in Sample A under negative bias over that in Sample B under positive bias, which is the current ratio of the SL with the MQWs over the SL with a single barrier. The lines with and without symbols are the dark current ratio at different temperatures and the photocurrent ratio, respectively. The photocurrent is measured at $15 \mathrm{~K}$ under $300-\mathrm{K}$ background radiation.

It is observed that the dark current from the SL with the MQWs is lower than that from only the MQWs. We attribute it to the asymmetrical graded barrier in Sample A. The neighboring barrier height of the SL $(\sim 235 \mathrm{meV})$ is higher than that of the MQWs $(\sim 170 \mathrm{meV})$ to result in the lower dark current from the SL. On the other hand, the photocurrent ratio is found to be higher than unity at bias lower than $0.3 \mathrm{~V}$. It is attributed to the broadband response under negative bias as described in Fig. 3. Also from Fig. 3, we observe that the photoresponse is not lowered by the additional MQWs. This result is consistent with the photocurrent ratio under low bias. As the bias is higher than $0.3 \mathrm{~V}$, the photocurrent ratio becomes lower than unity, and hence the photocurrent from the MQWs begins to dominate over that from the SL. Moreover, it also indicates that the photocurrent from the MQWs under negative bias is lower than that under 


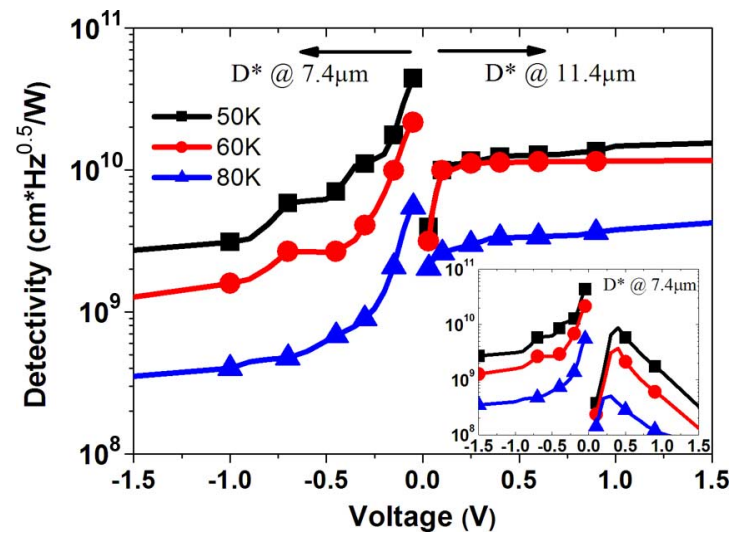

Fig. 5. Detectivity $\left(D^{*}\right)$ of Sample A versus voltage at 50,60 , and $80 \mathrm{~K}$. The corresponding $D^{*}$ wavelengths under positive and negative bias are 11.4 and $7.4 \mu \mathrm{m}$, respectively. The inset shows the comparison of $D^{*}$ at $7.4 \mu \mathrm{m}$ between Sample A and Sample B.

positive bias. It may be caused by the asymmetric graded barrier just like the reason of the lower dark current under negative bias.

In comparison with Sample B, Sample A also demonstrates the higher photocurrent and the lower dark current under the same applied bias. If we compare these two samples under the same electric field, the difference is more apparent. For the dark current, the causes of the higher value in Sample B are the lower barrier height $(\sim 202 \mathrm{meV})$ and the larger applied electric field under the same bias. On the other hand, although the photocurrent of the Sample A is also lowered by the higher graded barrier, it is still found to be higher than that of Sample B. We attribute this result to two factors. The first one is the photocurrent contribution of the MQWs as shown in the left inset of the Fig. 3. The second factor is the barrier scattering effect. Under low bias, the electron transport through the thin barrier is nearly ballistic. The equivalent single barrier width of Sample A is thinner than that of Sample B, which may lead to the higher photocurrent especially at very low bias. Although the current magnitude depends on the barrier thickness and barrier height simultaneously, it is our belief that the thinner barrier is more advantageous than the lower barrier for enhancing the photocurrent under low bias range because the photoelectrons have the higher energy.

\section{Detectivity}

From the results of responsivity and dark current, the detectivity $\left(D^{*}\right)$ at different temperatures is calculated and shown in Fig. 5. Here we choose $D^{*}$ at 11.4 and $7.4 \mu \mathrm{m}$ for the performance comparison under positive and negative bias, respectively, because the responses under different bias polarities come from the different structures. The different wavelengths are extracted from the peak positions of the spectrum in Fig. 3. We can find that the maximum $D^{*}$ value of Sample A appears at about $-0.1 \mathrm{~V}$. Despite the small responsivity at low bias, both the corresponding dark current and the noise gain are low enough to make $D^{*}$ increase as the bias magnitude decreases. Although the responsivity from the MQWs increases with the applied bias, the $D^{*}$ value of the MQWs saturates at high

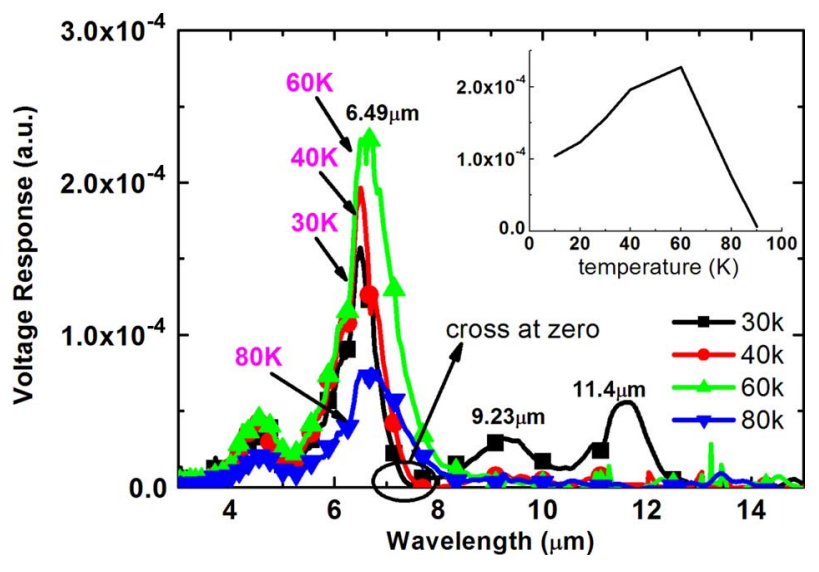

Fig. 6. PV response of Sample A at different temperatures. The inset shows the response intensity at $6.49 \mu \mathrm{m}$ versus temperature. We observe that the maximum of SL response appears at $60 \mathrm{~K}$, while the response from the MQWs disappears at high temperature. The cause of the zero-crossing at $7.5 \mu \mathrm{m}$ and the low intensity peak at $9.2 \mu \mathrm{m}$ is the opposite transport directions of the photoexcited electrons from the two structures.

positive bias and is still lower than the maximum value under negative bias.

In the inset of Fig. 5, the comparison of $D^{*}$ at $7.4 \mu \mathrm{m}$ between Sample A and B is shown. For the calculation of $D^{*}$, the noise current PSDs of Sample A and B are given by $4 e g_{n} I_{D}$ and $2 e I_{D}$, respectively. The $D^{*}$ of Sample A is better than that of Sample B due to the lower noise current PSD and the higher responsivity. Hence, it is concluded that the detector's performance is indeed improved by using the MQWs as the noise filter.

Nevertheless, the optimized operation range of Sample A under negative bias is quite small, because the $D^{*}$ value decreases rapidly as bias increasing. It would request more precise uniformity if we use this structure to fabricate the FPA.

Although we do not know the exact noise of our device, we measure the PV response at high temperature to prove that the noise is indeed reduced.

\section{E. Photovoltaic (PV) Response}

Ideally, the noise at zero bias is the thermal noise. Hence, Sample A's performance at zero bias is expected to be good. Fig. 6 shows the PV response of Sample A at different temperatures to demonstrate the zero-biased performance. Because we measure the PV spectrum by the voltage amplifier under open-circuit condition, the vertical axis in Fig. 6 is the relative voltage amplitude. Three peaks can be clearly distinguished from the spectrum. The $6.49-\mu \mathrm{m}$ peak is from the SL and the other two located at $9.2 \mu \mathrm{m}$ (from the bound-to-continuum transition) and $11.4 \mu \mathrm{m}$ (from the bound-to-bound transition) are from the MQWs. The peak position of the SL response under PV mode is slightly different from that in Fig. 3, because it shifts to longer wavelength when applied bias increases [14]. In particular, it is found that the SL can be operated under PV mode even at $80 \mathrm{~K}$, but the MQWs' PV response disappears once the temperature is higher than $30 \mathrm{~K}$. The SL has the better performance than the MQWs under PV mode, especially at high temperature. The inset in Fig. 6 is the plot of the SL's PV response intensity versus temperature. It first increases with temperature and then its maximum appears at $60 \mathrm{~K}$. The clear PV spectrum at high 


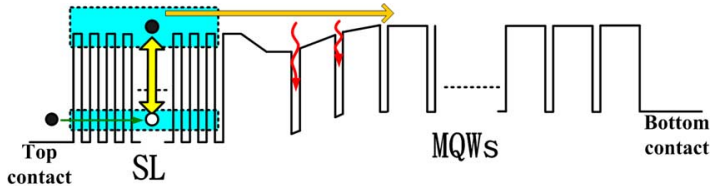

(a)

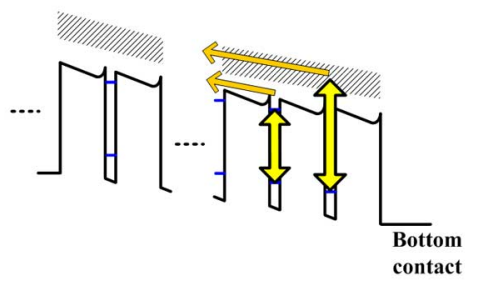

(b)

Fig. 7. Operational mechanism of Sample A under PV mode. (a) Photoexcited electrons from the SL will be captured by the MQWs to create the potential difference. (b) Potential difference caused by space charges inside the MQWs structure. The energy bending in the barrier is attributed to the dopant migration process. It is concluded that the transport directions of the photoelectrons from the SL and the MQWs are opposite.

temperature is the proof of the noise reduction. As mentioned in the end of Section III-B, the temperature variation of the response in Sample A will be studied in our future paper.

It is worth noting that the spectra in Figs. 3 and 6 are quite different at $8-10-\mu \mathrm{m}$ range. Especially in Fig. 6, only the $9.2-\mu \mathrm{m}$ peak from the MQWs is observed and its intensity is quite low. In addition, we also note that the PV spectrum crosses zero around $7.5 \mu \mathrm{m}$. These observations can be explained with the opposite transport directions of the photoelectrons from the SL and the MQWs. Fig. 7 shows the schematic PV-mode operational mechanism. The potential difference on each barrier in Fig. 7 is enlarged for emphasis. Under zero bias and the opencircuit condition, no electron can be collected by the contact and no photocurrent can thereby be generated. The first several quantum wells neighboring the SL work like traps to capture those photoelectrons from the SL as Fig. 7(a) shows. Because of the potential difference caused by those captured electrons, we can then measure the SL's PV voltage response. Besides, due to the dopant migration effect in the MQWs during the sample growth [21], an asymmetric energy band bending is created in the barriers. Therefore, the effective heights of two sides of the barrier are different, and make the electrons from the MQWs tend to move toward the left side rather than the right one as shown in Fig. 7(b). During the transport toward the top contact, some photoelectrons are recaptured and accumulated in the well. Those space charges produce another potential difference in the whole MQWs to block the electrons to move further towards the left side as shown in Fig. 7(b). Consequently, the voltage response from both the SL and the MQWs can be measured simultaneously under zero bias. However, the transport directions of the photoelectrons from these two structures are opposite to cause the opposite polarities of the voltage responses. The spectrum measured by FT-IR displays the total voltage quantity only and is unconcerned with the voltage polarity. We consider the $9.2-\mu \mathrm{m}$ peak is offset by the SL's response to result in the low intensity. Meanwhile, the zero-crossing point at $7.5 \mu \mathrm{m}$ is attributed to the equal opposite voltage contribution of the SL and the MQWs.

The zero-biased performance of Sample A is also comparable to other specific MQW structure for the PV applications [11]-[13].

\section{CONCLUSION}

In conclusion, a sample structure of a 15-period SL integrated with a 50-period MQWs is investigated. The functions of the MQWs are not only to act as a noise filter but also to add the response range. This structure demonstrates the broadband detection with spectral width $\Delta \lambda / \lambda>50 \%$ under low negative bias. In addition, from the results of current ratio, the photocurrent of the SL with the MQWs is higher than that of the MQWs only under low bias range due to the broadband photoresponse, while the dark current is lower by using the asymmetric graded barrier. Because of the low dark current and the low noise gain, the maximum $D^{*}$ appears at $-0.1 \mathrm{~V}$, which is even higher than the $D^{*}$ of the MQWs at high bias.

In comparison with the SL with a single barrier, this structure also demonstrates the better responsivity and $D^{*}$. For the higher response and photocurrent, we attribute those observations to the contribution of the MQWs and the ballistic transport across the equivalent thin barrier. Besides, because of the higher graded barrier, the lower dark current leads to the better $D^{*}$. Although the higher barrier also lowers the photocurrent, it is our belief that the thinner barrier is more advantageous than the lower barrier for enhancing the photocurrent at low bias.

In particular, this structure shows the good zero-biased performance and the SL's PV response can be observed even at $80 \mathrm{~K}$. From the observations of the low intensity peak at $9.2 \mu \mathrm{m}$ and the zero-crossing point at $7.5 \mu \mathrm{m}$ in the PV spectrum, we conclude that the transport directions of the photoelectrons from the SL and the MQWs under zero bias are opposite. From our experimental results, this device is appropriate for the operation at low bias and high temperature. However, the tradeoff is the small operation voltage range. It needs precise uniformity for the applications on the FPA.

\section{REFERENCES}

[1] J. Li, K. K. Choi, and D. C. Tsui, "Voltage-tunable quantum-well infrared photodetectors," Appl. Phys. Lett., vol. 86, pp. 2111141-211114-3, May 2005.

[2] A. Majumdar, K. K. Choi, L. P. Rokhinson, and D. C. Tsui, "Towards a voltage tunable quantum-well infrared photodetector," Appl. Phys. Lett., vol. 80, no. 4, pp. 538-540, Jan. 2002.

[3] M. Z. Tidrow, X. Jiang, S. S. Li, and K. Bacher, "A four-color quantum well infrared photodetector," Appl. Phys. Lett., vol. 74, no. 9, pp. 1335-1337, Mar. 1999.

[4] S. S. Li, S. H. Kim, J. H. Moon, and J. H. Lee, "A two-stack, multi-color quantum well infrared photodetector for mid and long-wavelength infrared detection," Infra. Phys. Technol., vol. 44, no. 5-6, pp. 235-241, Oct. 2003.

[5] M. Graf, G. Scalari, D. Hofstetter, J. Faist, H. Beere, E. Linfield, D. Ritchie, and G. Davies, "Terahertz range quantum well infrared photodetector," Appl. Phys. Lett., vol. 84, no. 4, pp. 475-477, Jan. 2004.

[6] H. C. Liu, C. Y. Song, A. J. SpringThorpe, and J. C. Cao, "Terahertz quantum-well photodetector," Appl. Phys. Lett., vol. 84, no. 20, pp. 4068-4070, May 2004.

[7] F. T. Vasko and E. P. O'Reilly, "THz photocurrent through an independently contacted three-level heterostructure," Semicond. Sci. Technol., vol. 19, pp. 558-560, Feb. 2004. 
[8] C. S. Wu, C. P. Wen, R. N. Sato, M. Hu, C. W. Tu, J. Zhang, L. D. Flesner, L. Pham, and P. S. Nayer, "Novel GaAs-AlGaAs multiquantum-well Schottky-junction device and its photovoltaic LWIR detection," IEEE Trans. Electron Devices., vol. 39, no. 2, pp. 234-241, Feb. 1992.

[9] Y. N. Soldatenko and F. T. Vasko, "Photovoltaic effect in coupled quantum wells under intersubband excitation," J. Appl. Phys., vol. 80, no. 7, pp. 4040-4044, Oct. 1996.

[10] Y. H. Wang, S. S. Li, and H. Pin, "Photovoltaic and photoconductive dual-mode operaion GaAs quantum-well infrared photodetector for 2-band detection," Appl. Phys. Lett., vol. 62, no. 1, pp. 93-95, Jan. 1993.

[11] H. Schneider, P. Koidl, C. Schonbein, S. Ehret, E. C. Larkins, and G. Bihlmann, "Capture dynamics and far-infrared response in photovoltaic quantum well intersubband photodetectors," Superlattices Microstruct., vol. 19, no. 4, pp. 347-356, 1996.

[12] O. Byungsung, J. W. Choe, M. H. Francombe, K. M. S. V. Bandara, E. Sorar, D. D. Coon, Y. F. Lin, and W. J. Takei, "Long-wavelength infrared detection in a photovoltaic-type superlattice structure," $J$. Vac. Sci. Technol. B, vol. 9, no. 3, pp. 1789-1793, May 1991.

[13] C. Schonbein, H. Schneider, G. Bihlmann, K. Schwarz, and P. Koidl, "A $10 \mu \mathrm{m} \mathrm{GaAs} / \mathrm{Al}_{x} \mathrm{Ga}_{1-x}$ As intersubband photodetector operating at zero bias voltage," Appl. Phys. Lett., vol. 68, no. 7, pp. 973-975, Feb. 1996.

[14] C. C. Chen, H. C. Chen, M. C. Hsu, W. H. Hsieh, C. H. Kuan, S. Y. Wang, and C. P. Lee, "Performance and application of a superlattice infrared photodetector with a blocking barrier," J. Appl. Phys., vol. 91, no. 3, pp. 943-948, Feb. 2003.

[15] C. C. Chen, H. C. Chen, C. H. Kuan, S. D. Lin, and C. P. Lee, "Multicolor infrared detection realized with two distinct superlattices seperated by a blocking barrier," Appl. Phys. Lett., vol. 80, no. 13, pp. 2251-2253, Apr. 2002.

[16] J. H. Lu, Y. Y. Yang, C. C. Chen, C. H. Kuan, H. T. Chen, and S. C. Lee, "Study of period number effect in the superlattice infrared photodetector," Infrared Phys. Technol., vol. 44, no. 5-6, pp. 399-409, Oct. 2003.

[17] K. K. Choi, The Physics of Quantum Well Infrared Photodetectors. River Edge, NJ: World Scientific, 1997, p. 294.

[18] A. S. Sedra and K. C. Smith, Microelectronic Circuits, 4th ed. Oxford, U.K.: Oxford Univ. Press, ch. 4.

[19] C. Y. Chen and C. H. Kuan, "Design and calibration of a noise measurement system," IEEE Trans. Instrum. Meas., vol. 49, no. 1, pp. 77-82, Feb. 2000.

[20] B. F. Levine, "Quantum-well infrared photodetector," J. Appl. Phys., vol. 74, no. 8, pp. R1-R81, Oct. 1993.

[21] K. K. Choi, The Physics of Quantum Well Infrared Photodetectors. River Edge, NJ: World Scientific, 1997, p. 304.

Jen-Hsiang Lu was born in Taiwan, R.O.C. He received the B.S. and M.S. degrees in electrical engineering from National Taiwan University, Taipei, Taiwan, R.O.C., in 2000 and 2002, respectively, where he is currently working toward the Ph.D. degree in electronics engineering.

His studies focused on the design and analysis of multicolor superlattice infrared photodetectors.

Kun-Jheng Wu was born in Taiwan, R.O.C. He received the B.S. degree in electrical engineering from National Chiao Tung University, Taiwan, R.O.C., in 2004. He is currently working toward the M.S. degree in electronics engineering at National Taiwan University, Taipei, Taiwan, R.O.C.

His studies focused on the fabrication of superlattice infrared photodetectors.
Kuang-Jou Hsieh was born in Taiwan, R.O.C. He received the B.S. degree in electrical engineering from National Cheng Kung University, Taiwan, R.O.C., in 2004. He is currently working toward the M.S. degree in electronics engineering at National Taiwan University, Taipei, Taiwan, R.O.C.

His studies focused on the fabrication of superlattice infrared photodetectors.

Chieh-Hsiung Kuan (M'95) was born in Taipei, Taiwan, R.O.C., in 1962. He received the B.S. degree in electrical engineering from National Taiwan University, Taipei, Taiwan, R.O.C., in 1985, and the M.S.A. and Ph.D. degrees in electrical engineering from Princeton University, Princeton, NJ, in 1990 and 1994, respectively. His Ph.D. dissertation involved dark current and noise characteristics of the infrared hot-electron transistors, in cooperation with the U.S Army Laboratory, Fort Monmouth, NJ.

In 1994, he joined the Department of Electrical Engineering, National Taiwan University, as an Associate Professor. His current research interests include infrared photodiodes for room-temperature operation, quantum-well infrared photodetectors and lasers, and topics on how to measure and suppress the noise indetectors.

Dr. Kuan is a member of Phi-Tau-Phi.

Juei-Yang Feng was born in Taiwan, R.O.C. He received the M.S. degrees in electrooptical engineering from National Sun Yat-Sen University, Taipei, Taiwan, R.O.C., in 2001, where he is currently working toward the Ph.D. degree in electrooptical engineering.

His studies focused on the MBE growth of electrooptical devices.

Tsong-Sheng Lay (M'93) was born in Taiwan, R.O.C., in 1964. He received the B.S. and M.S. degrees in electrical engineering from National Taiwan University, Taipei, Taiwan, R.O.C., and the M.A. and Ph.D. degrees in electrical engineering in 1993 and 1996, respectively, from Princeton University, Princeton, $\mathrm{NJ}$, where his research activities included magneto-transport and phase transitions of fractional quantum Hall states in coupled two-dimensional electron systems.

In 1996, he became a faculty member at National Sun Yat-Sen University,Taipei, Taiwan, R.O.C., where he is currently a Professor in the Institute of Electro-Optical Engineering. His research interests have focused on the optoelectronic devices of semiconductor quantum structures, molecular beam epitaxy, and the electrical and microstructure properties at high-k dielectric/semiconductor interfaces.

Dr. Lay is a member of the IEEE Lasers and Electro-Optics Society (LEOS) and the Materials Research Society.

Chen-Wei Yang was born in Taiwan, R.O.C. He received the B.S. and M.S degrees in electrophysics from National Chiao Tung University, Taiwan, R.O.C., in 2001 and 2003, respectively.

Since 2003, he has been with Opto Tech Corporation, Hsinchu, Taiwan, R.O.C., involved in developing high-efficiency LEDs.

Shun-Li Tu was born in Taiwan, R.O.C. He received the Ph. D. degree in electrooptical engineering from National Chiao Tung University, Taiwan, R.O.C., in 1990.

He is currently with Opto Tech Corporation, Hsinchu, Taiwan, R. O. C. involved in developing high-efficiency LEDs. 